\title{
Trapped lons Test Fundamental Particle Physics
}

\author{
New precision experiments using trapped molecular ions provide an alternative method for \\ determining if the electron has an electric dipole moment.
}

\section{by Nicholas R. Hutzler*}

$\mathrm{I}$ $\mathrm{t}$ is surprising that science cannot explain how matter was created after the big bang. All known physical processes leave the Universe with essentially equal amounts of matter and antimatter, so why is everything made of matter? This baryon asymmetry of the Universe (BAU) is one of the biggest mysteries facing modern physics and a driving force behind a wide range of efforts probing the frontier of particle physics. A new measurement from JILA in Colorado by the group led by Eric Cornell [1] explores this frontier with a tabletop system that uses trapped molecular ions to look for fundamental symmetry violations. The researchers specifically searched for the signature of an electric dipole moment in the electron. No signature was found, thus confirming previous experiments while at the same time constraining symmetry-breaking theories. The work demonstrates a novel and exciting approach to searching for new physics with precision measurements.

In 1967, Andrei Sakharov [2] studied the specific requirements for the existence of the BAU and found that a seemingly unrelated symmetry called time-reversal symmetry $(T)$ must be violated for matter to dominate antimatter. This realization has far-reaching experimental implications because signatures of $T$-violating physics can show up in a variety of seemingly unrelated places. For example, $T$-violating processes would cause fundamental particles such as electrons to have an electric dipole moment (EDM), which can be imagined classically as an uneven distribution of charge inside the particle. Since this charge imbalance can arise only from $T$-violating processes, and since $T$ violation commonly appears in models for new physics such as supersymmetry, searches for EDMs of fundamental particles are manifestly searches for new physics at high energies $[3,4]$.

One could search for an electron EDM, or eEDM, by placing an electron in an electric field, which would induce a torque on the particle if it has a dipole. This torque would be revealed in a precession, or wobbling, of the *Division of Physics, Mathematics, and Astronomy, California Insti-
tute of Technology, Pasadena, CA 91125, USA

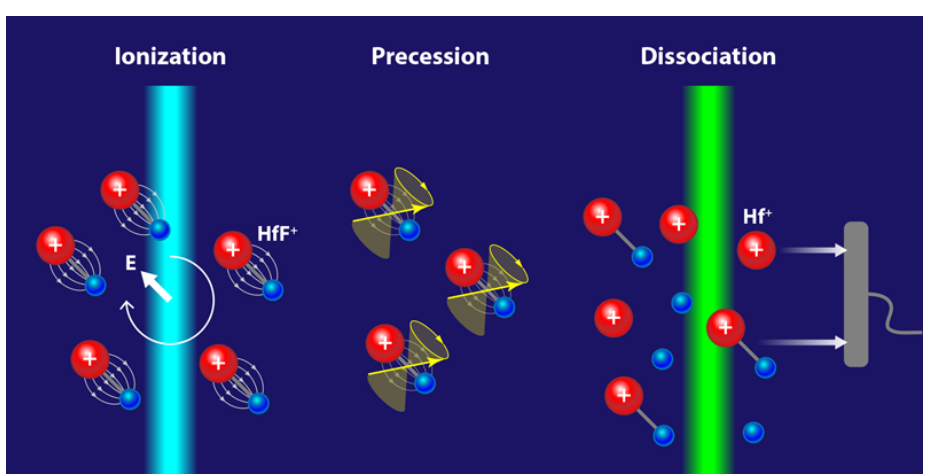

Figure 1: The JILA eEDM experiment [1] involves several steps. A sample of HfF molecules are ionized and confined in a cylindrical trap with a rotating electric field, $E$, which aligns the molecules (left). Each molecule has a strong internal electric field that can-if an eEDM exists-affect the spin precession of electrons around the molecule (middle). In the last step, a laser dissociates the molecules-depending on their precession angle-and an ion counter records the number of resulting $\mathrm{Hf}^{+}$ions. (APS/Alan Stonebraker)

electron's spin angular momentum. However, the internal electric fields inside some polar molecules are around a million times stronger than the external fields created in the lab [5]. Therefore, an experimentally superior approach is to select a heavy, polar molecule-heavy because the electrons should be moving relativistically-and search for a spin-precession signature in one of the outer (or valence) electrons. A common realization of such a measurement is to send a beam of neutral molecules through a vacuum chamber and probe the molecules spectroscopically with lasers or microwaves. The current best limit on the eEDM [6] and the previous few best limits [7] followed this general approach. However, molecular-beam experiments have a limitation-beams move, and eventually the molecules will leave the apparatus. Even fancy tricks like cryogenically cooling the molecules result in an observation time of just a few milliseconds, which means large numbers of molecules must be measured to build up an eEDM signal.

The JILA EDM experiment completely obliterates this limitation by trapping the molecules, increasing the observation 
time by a hundredfold, and therefore realizing a hundredfold increase in sensitivity per molecule. They start by using an ultraviolet laser to rip an electron from their molecule of choice, hafnium fluoride (HfF). The resulting $\mathrm{HfF}^{+}$ions are then trapped in rotating electric fields inside an ultrahigh vacuum chamber (Fig. 1). Since the electrostatic force is so strong, the ions are stuck rotating around the center of the ion trap, leaving plenty of time to perform an extremely precise measurement.

The team uses lasers to place the ionized molecules in a particular state in which the outermost electron has its spin pointing in a particular direction relative to the internal electric field of the molecule. After this state preparation, the experimenters allow the spins to precess freely for about one second. The rate of precession is controlled by several effects, including the presumed interaction of the eEDM with the molecule's electric field. At the end of the precession time, the researchers dissociate the molecules into atoms with a laser that can select a particular spin orientation. The atomic ions are then detected with an ion counter, yielding a measurement of the total precession angle, which is the experimental quantity of interest. Thanks to the relatively long trapping time, the precession angle is larger (and thus easier to measure) than in the previous experiments, which used molecular beams.

In a given experimental configuration, the precession angle depends on the eEDM along with a much larger collection of other contributions that are not necessarily of fundamental interest. The measurement is therefore repeated but with some experimental parameter reversed, for example, the orientation of the molecules relative to the ion trap fields. This parameter reversal would change the sign of the eEDM contribution to the precession angle, while not affecting other contributions. The measurement is then repeated again with some other parameter flipped, and so on. By measuring all possible combinations of nearly every experimental parameter, the experimenters isolate the eEDM signal from anything else. This isolation is robust enough to suppress systematic errors and uncertainties to a level that's below the uncertainty from finite statistics. In other words, with more ions and more data taking, the experiment can achieve higher sensitivity.

The JILA experiment did not see a signal of an eEDM, allowing them to set a limit that the eEDM must be no larger than about $10^{-28} e \mathrm{~cm}$ (where $e$ is the charge of one electron). This means that their experiment is sensitive to new physics at the few $\mathrm{TeV}$ scale, beyond even the reach of the LHC for $T$-violating physics that couples to the electron $[8,9]$. That is quite impressive for an experiment that would probably fit in the room where you are now sitting.

The JILA limit is within a factor of 2 of the currently lowest limit set by the Advanced Cold Molecule EDM (ACME) experiment a few years ago with a beam of neutral thorium monoxide (ThO) molecules [6]. Because the two experimental approaches are so different, they complement each other by having different sources of possible systematic errors to which the other may not be susceptible. This separateness will be critical in the event of a possible eEDM detection in the future. Both experiments are currently working on improved measurements which should be sensitive to $T$ violating physics at energy scales beyond $10 \mathrm{TeV}$. Regardless of whether the next measurements are null results, these tabletop molecule experiments will continue to push deeper into the frontiers of high-energy physics beyond the standard model.

This research is published in Physical Review Letters.

\section{REFERENCES}

[1] W. B. Cairncross, D. N. Gresh, M. Grau, K. C. Cossel, T. S. Roussy, Y. Ni, Y. Zhou, J. Ye, and E. A. Cornell, "Precision Measurement of the Electron's Electric Dipole Moment Using Trapped Molecular lons," Phys. Rev. Lett. 119, 153001 (2017).

[2] A. D. Sakharov, "Violation of CP Invariance, Asymmetry, and Baryon Asymmetry of the Universe," JETP Lett. 5, 27 (1967).

[3] D. DeMille, J. M. Doyle, and A. O. Sushkov, "Probing the Frontiers of Particle Physics with Tabletop-Scale Experiments," Science 357, 990 (2017).

[4] I. B. Khriplovich and S. K. Lamoreaux, CP Violation Without Strangeness: Electric Dipole Moments of Particles, Atoms, and Molecules (Springer-Verlag, Berlin, 1997).

[5] P. G. H. Sandars, "The Electric Dipole Moment of an Atom," Phys. Lett. 14, 194 (1965).

[6] J. Baron et al. (ACME Collaboration), "Order of Magnitude Smaller Limit on the Electric Dipole Moment of the Electron," Science 343, 269 (2014).

[7] J. J. Hudson, D. M. Kara, I. J. Smallman, B. E. Sauer, M. R. Tarbutt, and E. A. Hinds, "Improved Measurement of the Shape of the Electron," Nature 473, 493 (2011).

[8] J. Engel, M. J. Ramsey-Musolf, and U. van Kolck, "Electric Dipole Moments of Nucleons, Nuclei, and Atoms: The Standard Model and Beyond," Prog. Part. Nucl. Phys. 71, 21 (2013).

[9] J. L. Feng, "Naturalness and the Status of Supersymmetry," Annu. Rev. Nucl. Part. Sci. 63, 351 (2013).

10.1103/Physics. 10.111 